\title{
AXISYMMETRIC BESSEL LIGHT BEAMS
}

\author{
Pierre Hillion
}

Institut Henri Poincaré, 86 Bis Route de Croissy, 78110 Le Vésinet, France

\begin{abstract}
:
Bessel light beams satisfy a wave equation but, in addition, they are solutions of Maxwell's equations. The light power they convey is proportional to the square modulus of the electric field $\boldsymbol{E}$. In cylindrical coordinates, Bessel light beams depend on the polarization of $\boldsymbol{E}$ and, assuming an axisymmeteric beam, we give the ex-pressions of $\boldsymbol{E}$ when its polarization is, azi-muthal, radial and linear or circular. The energy flux carried by these beams is obtained from the time averaed Poynting vector.
\end{abstract}

\section{Keywords:}

Light beams, Bessel, Electric field, Polarization.

\section{INTRODUCTION}

Introduced by Durnin [1,2] as nondiffractive, Bessel beams have been the object of numerous works [3-6] and of some experiments with quasi Bessel beams (quasi since Bessel beams require an infinite amount of power). Only a few works were devoted to Besssel light beams $[8,9,10)$ which, in addition to satisfy a wave equation, are solutions of Maxwell's equations.

The light power conveyed by a Bessel light beam depends on the square modulus $|\mathbf{E}|^{2}$ of the electric field and, in a homogeneous, isotropic medium with permittivity $\varepsilon$ and permeability $\mu, \mathbf{E}$ satisfies the Helmholtz and divergence equations

$$
\left(\Delta+\mathrm{k}^{2}\right) \mathbf{E}=0 \quad, \quad \nabla \cdot \mathbf{E}=0 \quad, \mathrm{k}^{2}=\omega^{2} \varepsilon \mu / \mathrm{c}^{2}
$$

In cylindrical coordinates $\mathrm{r}, \phi, \mathrm{z}$, the Helmholtz equation is different for each coordinate of $\mathbf{E}$ and with $\nabla=1 / \mathrm{r} \partial_{\mathrm{r}}\left(\mathrm{r} \partial_{\mathrm{r}}\right)+1 / \mathrm{r}^{2} \partial_{\phi}{ }^{2}+\partial_{\mathrm{z}}{ }^{2}$, we get [11] for the components $\mathrm{E}_{\mathrm{r}}, \mathrm{E}_{\phi}, \mathrm{E}_{\mathrm{z}}$

$$
\begin{gathered}
\Delta \mathrm{E}_{\mathrm{r}}=\nabla^{2} \mathrm{E}_{\mathrm{r}}-1 / \mathrm{r}^{2} \mathrm{E}_{\mathrm{r}}-2 / \mathrm{r}^{2} \partial_{\phi} \mathrm{E}_{\phi} \\
\Delta \mathrm{E}_{\phi}=\nabla^{2} \mathrm{E}_{\phi}-1 / \mathrm{r}^{2} \mathrm{E}_{\phi}+2 / \mathrm{r}^{2} \partial_{\phi} \mathrm{E}_{\mathrm{r}} \\
\Delta \mathrm{E}_{\mathrm{z}}=\nabla^{2} \mathrm{E}_{\mathrm{z}}
\end{gathered}
$$

while the divergence equation becomes

$$
\left(\partial_{\mathrm{r}}+1 / \mathrm{r}\right) \mathrm{E}_{\mathrm{r}}+1 / \mathrm{r} \partial_{\phi} \mathrm{E}_{\phi}+\partial_{\mathrm{z}} \mathrm{E}_{\mathrm{z}}=0
$$

So, according to (2), Bessel light beams depend on the polarization of the electric field and, we shall consider successively azimuthal, radial, and linear or circular polarizations. In addition, Bessel light beams are supposed axisymmetric so that the electric field is only function of $r$ and $z$. Finaiiy we look for the solutions of the Helmholtz equation in the form

$$
\mathbf{E}(\mathrm{r}, \mathrm{z})=\mathrm{E}_{0} \exp \left(\mathrm{ik}_{\mathrm{z}} \mathrm{z}\right) \mathbf{E}(\mathrm{r})
$$

DOI : 10.14810/ijrap.2014.3203 
with the constant amplitude $\mathrm{E}_{0}$.

The energy flux carried by axisymmetric Bessel light beams is obtained from the time averaged Poynting vector.

\section{ELECTRIC FIELD POLARIZATION}

The polarization of the electric field $\mathbf{E}$ in Bessel light beams is carefully discussed in $[8,12]$. We follow closely [12] and we impose $\partial_{\phi} \mathbf{E}=0$ to satisfy the axisymmetric condition.

2.1 The azimuthal polarization is characterized by $E_{r}=E_{z}=0$ so that according to (2) and since $\partial_{\phi} E_{\phi}=0$, the Helmholz equation satisfied by $E_{\phi}$ is

$$
\left(\partial_{\mathrm{r}}^{2}+1 / \mathrm{r} \partial_{\mathrm{r}}-1 / \mathrm{r}^{2}+\partial_{\mathrm{z}}^{2}+\mathrm{k}^{2}\right) \underset{2}{\mathrm{E}_{\phi}(\mathrm{r}, \mathrm{z})=0}
$$

with the solutions in which $\mathrm{k}_{\mathrm{r}}^{2}+\mathrm{k}_{\mathrm{z}}^{2}=\mathrm{k}^{2}$

$$
\mathrm{E}_{\phi}(\mathrm{r}, \mathrm{z})=\mathrm{E}_{0} \mathrm{~J}_{1}\left(\mathrm{k}_{\mathrm{r}} \mathrm{r}\right) \exp \left(\mathrm{ik}_{\mathrm{z}} \mathrm{z}\right)
$$

$J_{1}$ is the Bessel function of the first kind of order one and the divergence equation (2a) is trivially sa-tisfied.

2.2 For the radial polarization, only the component $E_{\phi}$ is null. The component $E_{r}$, according to (2) and taking into account $\partial_{\phi} \mathrm{E}_{\mathrm{r}}=0$, satisfies the Helmholtz equation (4) so that

$$
\mathrm{E}_{\mathrm{r}}(\mathrm{r}, \mathrm{z})=\mathrm{E}_{0} \mathrm{~J}_{1}\left(\mathrm{k}_{\mathrm{r}} \mathrm{r}\right) \exp \left(\mathrm{ik}_{\mathrm{z}} \mathrm{z}\right)
$$

Now, the component $E_{z}$ still from (2) and from $\partial_{\phi} E_{z}=0$, is solution of the Helmholtz equation

$$
\left(\partial_{\mathrm{r}}^{2}+1 / \mathrm{r} \partial_{\mathrm{r}}+\partial_{\mathrm{z}}^{2}+\mathrm{k}^{2}\right) \mathrm{E}_{\mathrm{z}}(\mathrm{r}, \mathrm{z})=0
$$

with the solutions

$$
\mathrm{E}_{\mathrm{z}}(\mathrm{r}, \mathrm{z})=-\mathrm{iE}_{0} \mathrm{k}_{\mathrm{r}} / \mathrm{k}_{\mathrm{z}} \mathrm{J}_{0}\left(\mathrm{k}_{\mathrm{r}} \mathrm{r}\right) \exp \left(\mathrm{ik}_{\mathrm{z}} \mathrm{z}\right)
$$

in which $\mathrm{J}_{0}$ is the Bessel function of the first kind of order zero.

And using the relation $\left(\partial_{\mathrm{r}}+1 / \mathrm{r}\right) \mathrm{J}_{1}\left(\mathrm{k}_{\mathrm{r}} \mathrm{r}\right)=\mathrm{k}_{\mathrm{r}} \mathrm{J}_{0}\left(\mathrm{k}_{\mathrm{r}} \mathrm{r}\right)$, it is checked at once that the divergence equation (2) is satisfied.

2.3 For linear and circular polarizations, the electric field, in cartesian coordinates is obtained from Eq.(12) of [12] in the form

$$
\mathrm{E}(\mathrm{r}, \mathrm{z})=\mathrm{E}_{0} \exp \left(\mathrm{ik}_{\mathrm{z}} \mathrm{z}\right)\left[\left(\alpha \mathrm{u}_{\mathrm{x}}+\beta \mathrm{u}_{\mathrm{y}}\right) \mathrm{J}_{0}\left(\mathrm{k}_{\mathrm{r}} \mathrm{r}\right)+\mathrm{ik}_{\mathrm{r}} / 2 \mathrm{k}_{\mathrm{z}}\left\{(\alpha+\mathrm{i} \beta) \exp (-\mathrm{i} \phi) \mathrm{J}_{-1}\left(\mathrm{k}_{\mathrm{r}} \mathrm{r}\right)+(\alpha-\mathrm{i} \beta) \exp (\mathrm{i} \phi)\right.\right.
$$
$\left.\left.\mathrm{J}_{1}\left(\mathrm{k}_{\mathrm{r}} \mathrm{r}\right)\right\} \mathrm{u}_{\mathrm{z}}\right]$

$\mathrm{u}_{\mathrm{x}}, \mathrm{u}_{\mathrm{y}}, \mathrm{u}_{\mathrm{z}}$ are the unit vectors in cartesian coordinates Then, since $\mathrm{J}_{-1}=-\mathrm{J}_{1}$, we get from (9)

$$
\begin{aligned}
& \mathrm{E}_{\mathrm{x}}(\mathrm{r}, \mathrm{z})=\alpha \mathrm{E}_{0} \exp \left(\mathrm{ik}_{\mathrm{z}} \mathrm{z}\right) \mathrm{J}_{0}\left(\mathrm{k}_{\mathrm{r}} \mathrm{r}\right) \\
& \mathrm{E}_{\mathrm{y}}(\mathrm{r}, \mathrm{z})=\beta \mathrm{E}_{0} \exp \left(\mathrm{ik}_{\mathrm{z}} \mathrm{z}\right) \mathrm{J}_{0}\left(\mathrm{k}_{\mathrm{r}} \mathrm{r}\right) \\
& \left.\mathrm{E}_{\mathrm{z}}(\mathrm{r}, \mathrm{z})=-\mathrm{ik}_{\mathrm{r}} / \mathrm{k}_{\mathrm{z}}(\alpha \cos \phi+\beta \sin \phi) \mathrm{E}_{0} \exp \left(\mathrm{ik}_{\mathrm{z}} \mathrm{z}\right) \mathrm{J}_{1}\left(\mathrm{k}_{\mathrm{r}} \mathrm{r}\right)\right]
\end{aligned}
$$


For a linear polarization the parameters $\alpha, \beta$ are both real while for right and left circular polarizations, they satisfy $\beta=i \alpha$ and $\beta=-i \alpha$ respectively.

In these last expressions $r=\left(x^{2}+y^{2}\right)^{1 / 2}$, and a simple calculation proves that the divergence equation

$$
\partial_{\mathrm{x}} \mathrm{E}_{\mathrm{x}}+\partial_{\mathrm{y}} \mathrm{E}_{\mathrm{y}}+\partial_{\mathrm{z}} \mathrm{E}_{\mathrm{z}}=0
$$

is fulfilled.

\section{BESSEL LIGHT BEAMS}

These results are summarized in the following table leaving aside $\mathrm{E}_{0} \exp \left(\mathrm{ik}_{\mathrm{z}} \mathrm{z}\right)$

Table 1

\begin{tabular}{llll} 
Polarization & $:$ & electric field E(r) \\
Azimuthal & $:$ & $\mathrm{E}_{\mathrm{r}}(\mathrm{r})=\mathrm{E}_{\mathrm{z}}(\mathrm{r})=0, \quad \mathrm{E}_{\phi}(\mathrm{r}) \approx \mathrm{J}_{1}\left(\mathrm{k}_{\mathrm{r}} \mathrm{r}\right)$ \\
Radial & $:$ & $\mathrm{E}_{\phi}(\mathrm{r}) \approx 0 \quad \mathrm{E}_{\mathrm{r}}(\mathrm{r}) \approx \mathrm{J}_{1}\left(\mathrm{k}_{\mathrm{r}} \mathrm{r}\right), \mathrm{E}_{\mathrm{z}}(\mathrm{r}) \approx \mathrm{J}_{0}\left(\mathrm{k}_{\mathrm{r}} \mathrm{r}\right)$ \\
Linear & $:$ & $\mathrm{E}_{\mathrm{x}}(\mathrm{r}) \approx \alpha \mathrm{J}_{0}\left(\mathrm{k}_{\mathrm{r}} \mathrm{r}\right), \quad \mathrm{E}_{\mathrm{y}}(\mathrm{r}) \approx \beta \mathrm{J}_{0}\left(\mathrm{k}_{\mathrm{r}} \mathrm{r}\right), \quad \mathrm{E}_{\mathrm{z}}(\mathrm{r})=-i \alpha \mathrm{k}_{\mathrm{r}} / \mathrm{k}_{\mathrm{z}}(\alpha \cos \phi+$ \\
& & \multicolumn{2}{c}{$\beta \sin \phi) \mathrm{J}_{1}\left(\mathrm{k}_{\mathrm{r}} \mathrm{r}\right)}$.
\end{tabular}

This table shows how the structure of Bessel light beams depends on the electric field polarization and, in any case, this structure reduces to that of conventional Bessel beams.

The importance of Bessel light beams lies in the energy flux they carry which may be obtained from the time averaged Poynting vector $[12,13]$

$$
\mathbf{S}=1 / 4 i \omega \mu\left[\mathbf{E} \wedge \operatorname{curl} \mathbf{E}^{*}+\{\mathrm{cc}\}\right]
$$

the asterisk denotes the complex conjugation and $\{\mathrm{cc}\}=\mathrm{E}^{*} \wedge \mathrm{curl} \mathbf{E}$.

For an axisymmetric beam $\partial_{\phi} \mathbf{E}=0$ and the components of curl $\mathbf{E}^{*}$ are

$$
\begin{gathered}
\left(\operatorname{curl} \mathbf{E}^{*}\right)_{\mathrm{r}}=-\partial_{\mathrm{z}} \mathrm{E}_{\phi}{ }^{*} \\
\left(\operatorname{curl} \mathbf{E}^{*}\right)_{\phi}=\partial_{\mathrm{z}} \mathrm{E}_{\mathrm{r}}^{*}-\partial_{\mathrm{r}} \mathrm{E}_{\mathrm{z}}^{*} \\
\left(\operatorname{curl} \mathbf{E}^{*}\right)_{\mathrm{z}}=\left(\partial_{\mathrm{r}}+1 / \mathrm{r}\right) \mathrm{E}_{\phi}{ }^{*}
\end{gathered}
$$

so that

$$
\begin{aligned}
\left(\mathbf{E} \wedge \operatorname{curl} \mathbf{E}^{*}\right)_{\mathrm{r}} & =\mathrm{E}_{\phi}\left(\operatorname{curl} \mathbf{E}^{*}\right)_{\mathrm{z}}-\mathrm{E}_{\mathrm{z}}\left(\operatorname{curl} \mathbf{E}^{*}\right)_{\phi} \\
& =\mathrm{E}_{\phi}\left(\partial_{\mathrm{r}}+1 / \mathrm{r}\right) \mathrm{E}_{\mathrm{z}}^{*}-\mathrm{E}_{\mathrm{z}}\left(\partial_{\mathrm{z}} \mathrm{E}_{\mathrm{r}}^{*}-\partial_{\mathrm{r}} \mathrm{E}_{\mathrm{z}}^{*}\right) \\
\left(\mathbf{E} \wedge \operatorname{curl} \mathbf{E}^{*}\right)_{\phi} & =\mathrm{E}_{\mathrm{z}}\left(\operatorname{curl} \mathbf{E}^{*}\right)_{\mathrm{r}}-\mathrm{E}_{\mathrm{r}}\left(\operatorname{curl} \mathbf{E}^{*}\right)_{\mathrm{z}} \\
& 3 \\
& \left.=-\mathrm{E}_{\mathrm{z}} \partial_{\mathrm{z}} \mathrm{E}_{\phi}^{*}-\mathrm{E}_{\mathrm{r}} \partial_{\mathrm{r}}+1 / \mathrm{r}\right) \mathrm{E}_{\phi}^{*} \\
\left(\mathbf{E} \wedge \operatorname{curl} \mathbf{E}^{*}\right)_{\mathrm{z}} & =\mathrm{E}_{\mathrm{r}}\left(\operatorname{curl} \mathbf{E}^{*}\right)_{\phi}-\mathrm{E}_{\phi}\left(\operatorname{curl} \mathbf{E}^{*}\right)_{\mathrm{r}} \\
& =\mathrm{E}_{\mathrm{r}}\left(\partial_{\mathrm{z}} \mathrm{E}_{\mathrm{r}}^{*}-\partial_{\mathrm{r}} \mathrm{E}_{\mathrm{z}}^{*}\right)-\mathrm{E}_{\phi} \partial_{\mathrm{z}} \mathrm{E}_{\phi}^{*}
\end{aligned}
$$

Then, for azimuthal polarization $\mathrm{E}_{\mathrm{r}}=\mathrm{E}_{\mathrm{z}}=0$ and taking into account (5) and (14) we get from (12) for the components of the Poynting vector

$$
\mathrm{S}_{\mathrm{r}}=\mathrm{S}_{\phi}=0 \quad \mathrm{~S}_{\mathrm{z}}=\left[\left|\mathrm{E}_{0}\right|^{2} \mathrm{k}_{\mathrm{z}} / 2 \omega \mu\right]\left|\mathrm{J}_{1}\left(\mathrm{k}_{\mathrm{r}} \mathrm{r}\right)\right|^{2}
$$


A result also valid for the radial polarization since $\mathrm{E}_{\phi}=0$ and since according to (8)

$$
\mathrm{E}_{\mathrm{z}}\left(\partial_{\mathrm{z}} \mathrm{E}_{\mathrm{r}}^{*}-\partial_{\mathrm{r}} \mathrm{E}_{\mathrm{z}}^{*}\right)+\{\mathrm{cc}\}=0
$$

So, from a Poynting point of view, taking (15) into account, an axisymmetric Bessel light beam could be characterized by the Bessel function $\mathrm{J}_{1}$ and not by $\mathrm{J}_{0}$ as it is usually assumed.We leave aside the most intricate Poynting vector for linear and circular polarizations also discussed in [12].

\section{CONCLUSION}

Works on conventional Bessel beams with $\mathrm{J}_{0}$ are flourishing [1-6] while those on Bessel light beams are few. Nevertheless, starting with Stratton [14] (see [15]) some authors have considered non-diffractive higher order Bessel beams with $\mathrm{J}_{\mathrm{m}} \mathrm{m}$ integer. But although these fields satisfy the Hel-mholtz equation, they do not necessary satisfy the Maxwell's equations. In [8], some of the results given here, are obtained for a vector potential, written as a superposition of the solutions to the vector Helmholtz equation, nevertheless this work [8] is not referenced in [15].

Remark : Bessel light beams depend on a conicity angle and when this angle is small $(\leq \mathrm{qq}$ degrees) their z-component becomes negligible and they can be described as a field with the $r$ component pro-portional to $\mathrm{J}_{0}$.

The results obtained here may be generalized in two directions. In [16], a scalar, axisymmetric, pulsed Bessel beam has the representation

$$
\psi(\mathrm{r}, \mathrm{z}, \omega)=\mathrm{J}_{0}\left(\mathrm{r} / \mathrm{r}_{0}\right) \exp [\mathrm{i} \beta(\mathrm{z}) \mathrm{z}] \mathrm{f}\left(\omega-\omega_{0}\right)
$$

$r_{0}$ is constant, $\mathrm{f}\left(\omega-\omega_{0}\right)$ is the spectral distribution with $\mathrm{f}\left(\omega_{0}\right)$ at $\mathrm{z}=0$ and

$$
\beta(z)=\beta_{0}+\sum_{1}^{\infty} \beta_{m} / m !\left(\omega-\omega_{0}\right)^{m}
$$

Then, assuming

$$
f\left(\omega-\omega_{0}\right)=1 / \sqrt{ } 2 \pi T_{0} \exp \left[T_{0}^{3}\left(\omega-\omega_{0}\right)^{3} / 3\right]
$$

the inverse Fourier transform of (17) supplies the Airy Bessel pulsed beam $\Psi(\mathrm{r}, \mathrm{z}, \mathrm{t})$.

This technique could be applied to a Bessel light beam with azimuthal polarization just by changing $\mathbf{J}_{0}$ into $\mathbf{J}_{1}$ (see Table 1 of Sec.3) and giving an Airy-Bessel pulsed light beam.

Now, using Hertzian vector potentials of electric and magnetic types $\Pi_{e}, \Pi_{m}$ solutions of the Helmholtz equation $\left(\Delta+k^{2}\right) \Pi=0$, we get [16] for the electric and magnetic fields

$$
\begin{array}{llll}
\mathbf{E}_{\mathrm{e}}=\nabla \wedge \nabla \wedge \boldsymbol{\Pi}_{\mathrm{e}}=\nabla \nabla \cdot \boldsymbol{\Pi}_{\mathrm{e}}+\mathrm{k}^{2} \boldsymbol{\Pi}_{\mathrm{e}} & , \quad \mathbf{E}_{\mathrm{m}}=-\mathrm{i} \mu_{0} \nabla \wedge \boldsymbol{\Pi}_{\mathrm{m}} & \text { a) } \\
\mathbf{E}_{\mathrm{m}}=\nabla \wedge \nabla \wedge \boldsymbol{\Pi}_{\mathrm{m}}=\nabla \nabla \cdot \boldsymbol{\Pi}_{\mathrm{m}}+\mathrm{k}^{2} \boldsymbol{\Pi}_{\mathrm{m}} \quad, \quad \mathbf{H}_{\mathrm{m}}=\mathrm{i} \mu_{0} \nabla \wedge \boldsymbol{\Pi}_{\mathrm{m}} & \text { b) }
\end{array}
$$

This technique is used in [17] to get TE, TM Bessel beams.

Let us now use cylindrical coordinates, then with a judicious choice of $\Pi$, we get further expressions of Bessel light beams. Suppose first $\Pi_{r}=\Pi_{\phi}=0$, then we get [18] from (19a,b) the following expressions of the electric field

$$
\mathrm{E}_{\mathrm{r}}=-\mathrm{i} \omega \mu / \mathrm{r} \partial_{\phi} \Pi_{\mathrm{z}} \quad, \quad \mathrm{E}_{\mathrm{r}}=\partial_{\mathrm{r}} \partial_{\mathrm{z}} \Pi_{\mathrm{z}}
$$


International Journal of Recent advances in Physics (IJRAP) Vol.3, No.2, May 2014

$$
\begin{array}{llll}
\mathrm{E}_{\phi}=\mathrm{i} \omega \mu \partial_{\mathrm{r}} \Pi_{\mathrm{z}} & & , & \mathrm{E}_{\phi}=1 / \mathrm{r} \partial_{\phi} \partial_{\mathrm{z}} \Pi_{\mathrm{z}} \\
\mathrm{E}_{\mathrm{z}}=0 & (20 \mathrm{~b}) & , & \mathrm{E}_{\mathrm{z}}=\left(\mathrm{k}^{2}+\partial_{\mathrm{z}}{ }^{2}\right) \Pi_{z}
\end{array}
$$

$\mathrm{E}_{\mathrm{z}}=\left(\mathrm{k}^{2}+\partial_{\mathrm{z}}^{2}\right) \Pi_{\mathrm{z}} \quad(20 \mathrm{a})$

But, $\Pi_{z}$ is solution of the wave equation (7) so that $\left(A_{z}\right.$ is a constant amplitude)

$$
\left.\Pi_{\mathrm{z}}=\mathrm{A}_{\mathrm{z}} \exp \left(\mathrm{ik}_{\mathrm{z}} \mathrm{z}\right) \mathrm{J}_{0}\left(\mathrm{k}_{\mathrm{r}} \mathrm{r}\right)\right] \quad, \quad \mathrm{k}_{\mathrm{r}}^{2}+\mathrm{k}_{\mathrm{z}}^{2}=\mathrm{k}^{2}
$$

Substituting (21) into (20a,b) and using the relations

$$
\partial_{\mathrm{r}} \mathrm{J}_{0}\left(\mathrm{k}_{\mathrm{r}} \mathrm{r}\right)=-\mathrm{k}_{\mathrm{r}} \mathrm{J}_{1}\left(\mathrm{k}_{\mathrm{r}} \mathrm{r}\right) \quad, \quad\left(\partial_{\mathrm{r}}+1 / \mathrm{r}\right) \mathrm{J}_{1}\left(\mathrm{k}_{\mathrm{r}} \mathrm{r}\right)=\mathrm{k}_{\mathrm{r}} \mathrm{J}_{0}\left(\mathrm{k}_{\mathrm{r}} \mathrm{r}\right)
$$

give

$$
\begin{aligned}
& \left.\mathrm{E}_{\mathrm{r}}=-\mathrm{ik}_{\mathrm{r}} \mathrm{k}_{\mathrm{z}} \mathrm{A}_{\mathrm{z}} \exp \left(\mathrm{ik}_{\mathrm{z}} \mathrm{z}\right) \mathrm{J}_{1}\left(\mathrm{k}_{\mathrm{r}} \mathrm{r}\right)\right] \quad, \quad \mathrm{E}_{\mathrm{r}}=0 \\
& \left.\mathrm{E}_{\phi}=0 \quad, \quad \mathrm{E}_{\phi}=i \omega \mu \mathrm{k}_{\mathrm{r}} \mathrm{A}_{\mathrm{z}} \exp \left(\mathrm{ik}_{\mathrm{z}} \mathrm{z}\right) \mathrm{J}_{1}\left(\mathrm{k}_{\mathrm{r}} \mathrm{r}\right)\right] \\
& \left.\mathrm{E}_{\mathrm{z}}=\mathrm{k}_{\mathrm{r}}^{2} \mathrm{~A}_{\mathrm{z}} \exp \left(\mathrm{ik}_{\mathrm{z}} \mathrm{z}\right) \mathrm{J}_{\mathrm{o}}\left(\mathrm{k}_{\mathrm{r}} \mathrm{r}\right)\right] \quad(23 \mathrm{a}), \quad \mathrm{E}_{\mathrm{z}}=0 \\
& 4
\end{aligned}
$$

Similarly for $\Pi_{\phi}=\Pi_{z}=0$, we get from $(19 \mathrm{a}, \mathrm{b})$

$$
\begin{array}{llll}
\mathrm{E}_{\mathrm{r}}=0 & & , & \mathrm{E}_{\mathrm{r}}=\left[\mathrm{k}^{2}+\partial_{\mathrm{r}}\left(\partial_{\mathrm{r}}+1 / \mathrm{r}\right)\right] \Pi_{\mathrm{r}} \\
\mathrm{E}_{\phi}=-\mathrm{i} \omega \mu \partial_{\mathrm{z}} \Pi_{\mathrm{r}} & & , & \mathrm{E}_{\phi}=1 / \mathrm{r} \partial_{\phi} \partial_{\mathrm{r}}\left(\mathrm{r} \Pi_{\mathrm{r}}\right) \\
\mathrm{E}_{\mathrm{z}}=\mathrm{i} \omega \mu / \mathrm{r} \partial_{\phi} \Pi_{\mathrm{r}} & (24 \mathrm{~b}) \quad, & \mathrm{E}_{\mathrm{z}}=1 / \mathrm{r} \partial_{\mathrm{z}} \partial_{\mathrm{r}}\left(\mathrm{r} \Pi_{\mathrm{r}}\right)
\end{array}
$$

with $\Pi_{\mathrm{r}}$ solution of the wave equation (4) so that

$$
\left.\Pi_{\mathrm{r}}=\mathrm{A}_{\mathrm{r}} \exp \left(\mathrm{ik}_{\mathrm{z}} \mathrm{z}\right) \mathrm{J}_{1}\left(\mathrm{k}_{\mathrm{r}} \mathrm{r}\right)\right]
$$

Substituting (25) into (24a,b) and using (22) give

$$
\begin{aligned}
& \mathrm{E}_{\mathrm{r}}=\mathrm{k}_{\mathrm{z}}^{2} \mathrm{~A}_{\mathrm{r}} \exp \left(\mathrm{ik}_{\mathrm{z}} \mathrm{z}\right) \mathrm{J}_{1}\left(\mathrm{k}_{\mathrm{r}} \mathrm{r}\right) \quad, \quad \mathrm{E}_{\mathrm{r}}=0 \\
& \mathrm{E}_{\phi}=0 \quad, \quad \mathrm{E}_{\phi}=\mathrm{i} \omega \mu \mathrm{k}_{\mathrm{z}} \mathrm{A}_{\mathrm{z}} \exp \left(\mathrm{ik}_{\mathrm{z}} \mathrm{z}\right) \mathrm{J}_{1}\left(\mathrm{k}_{\mathrm{r}} \mathrm{r}\right) \\
& \left.\mathrm{E}_{\mathrm{z}}=\mathrm{ik}_{\mathrm{z}} \mathrm{k}_{\mathrm{r}} \mathrm{A}_{\mathrm{r}} \exp \left(\mathrm{ik}_{\mathrm{z}} \mathrm{z}\right) \mathrm{J}_{0}\left(\mathrm{k}_{\mathrm{r}} \mathrm{r}\right)\right] \quad(26 \mathrm{a}) \quad, \quad \mathrm{E}_{\mathrm{z}}=0
\end{aligned}
$$

Finally for $\Pi_{z}=\Pi_{r}=0$, it comes [18]

$$
\begin{array}{llll}
\mathrm{E}_{\mathrm{r}}=\mathrm{i} \omega \mu \partial_{\mathrm{z}} \Pi_{\phi} & & , & \mathrm{E}_{\mathrm{r}}=1 / \mathrm{r} \partial_{\mathrm{r}} \partial_{\phi} \Pi_{\phi} \\
\mathrm{E}_{\phi}=0 & & , & \mathrm{E}_{\phi}=\left(\mathrm{k}^{2}+1 / \mathrm{r}^{2} \partial_{\phi}^{2}\right) \Pi_{\phi} \\
\mathrm{E}_{\mathrm{z}}=\mathrm{i} \omega \mu / \mathrm{r} \partial_{\mathrm{r}}\left(\mathrm{r} \Pi_{\phi}\right) & (27 \mathrm{a}) & , & \mathrm{E}_{\mathrm{z}}=1 / \mathrm{r} \partial_{\mathrm{z}} \partial_{\phi} \Pi_{\phi}
\end{array}
$$

In which $\Pi_{\phi}$ is solution of the wave equation (4) so that

$$
\left.\Pi_{\phi}=\mathrm{A}_{\phi} \exp \left(\mathrm{ik}_{\mathrm{z}} \mathrm{z}\right) \mathrm{J}_{1}\left(\mathrm{k}_{\mathrm{r}} \mathrm{r}\right)\right]
$$

Substituting (28) into (27a,b) and still using (22) give

$$
\begin{array}{lrlrl}
\mathrm{E}_{\mathrm{r}}=0 & , & \left.\mathrm{E}_{\mathrm{r}}=\omega \mu \mathrm{k}_{\mathrm{z}} \mathrm{A}_{\phi} \exp \left(\mathrm{ik}_{\mathrm{z}} \mathrm{z}\right) \mathrm{J}_{1}\left(\mathrm{k}_{\mathrm{r}} \mathrm{r}\right)\right] \\
\left.\mathrm{E}_{\phi}=\mathrm{k}^{2} \mathrm{~A}_{\phi} \exp \left(\mathrm{ik}_{\mathrm{z}} \mathrm{z}\right) \mathrm{J}_{1}\left(\mathrm{k}_{\mathrm{r}} \mathrm{r}\right)\right] & , & \mathrm{E}_{\phi}=0 \\
\mathrm{E}_{\mathrm{z}}=0 & (29 \mathrm{a}) & , & \left.\mathrm{E}_{\mathrm{z}}=\mathrm{i} \omega \mu \mathrm{k}_{\mathrm{r}} \mathrm{A}_{\phi} \exp \left(\mathrm{ik}_{\mathrm{z}} \mathrm{z}\right) \mathrm{J}_{0}\left(\mathrm{k}_{\mathrm{r}} \mathrm{r}\right)\right]
\end{array}
$$

Comparing these results with those of the Table 1 in Sec.3 shows that (23b), (26b), (29a) correspond to Bessel light beams with azimuthal polarization while for (23a), (26a), (29b) the polarization is radial. We may now combine these fields to get Bessel light beams with a more 
elaborated sructure. For instance, substituting (26a) and (23a) on one hand and (23b) nd (26b) on the other hand gives

and

$$
\begin{aligned}
& \left.\mathrm{E}_{\mathrm{r}}=\left(-i \mathrm{k}_{\mathrm{r}} \mathrm{k}_{\mathrm{z}} \mathrm{A}_{\mathrm{z}}+\mathrm{k}_{\mathrm{z}}^{2} \mathrm{~A}_{\mathrm{r}}\right) \exp \left(i \mathrm{k}_{\mathrm{z}} \mathrm{z}\right) \mathrm{J}_{1}\left(\mathrm{k}_{\mathrm{r}} \mathrm{r}\right)\right] \\
& \mathrm{E}_{\phi}=0 \\
& \left.\mathrm{E}_{\mathrm{z}}=\left(\mathrm{k}_{\mathrm{r}}^{2} \mathrm{~A}_{\mathrm{z}}+\mathrm{ik}_{\mathrm{z}} \mathrm{k}_{\mathrm{r}} \mathrm{A}_{\mathrm{r}}\right) \exp \left(\mathrm{ik}_{\mathrm{z}} \mathrm{z}\right) \mathrm{J}_{\mathrm{o}}\left(\mathrm{k}_{\mathrm{r}} \mathrm{r}\right)\right]
\end{aligned}
$$

$$
\begin{aligned}
& \mathrm{E}_{\mathrm{r}}=0 \\
& \left.\mathrm{E}_{\phi}=\left(i \omega \mu \mathrm{k}_{\mathrm{r}} \mathrm{A}_{\mathrm{z}}+\mathrm{i} \omega \mu \mathrm{k}_{\mathrm{z}} \mathrm{A}_{\mathrm{r}}\right) \exp \left(\mathrm{ik}_{\mathrm{z}} \mathrm{z}\right) \mathrm{J}_{1}\left(\mathrm{k}_{\mathrm{r}} \mathrm{r}\right)\right] \\
& \mathrm{E}_{\mathrm{z}}=0
\end{aligned}
$$

The polarization radial in (30a) is azimuthal in (30b). Similarly with (26a), (29a),(26b), (29b),

$$
\begin{aligned}
& \mathrm{E}_{\mathrm{r}}=\mathrm{k}_{\mathrm{z}}^{2} \mathrm{~A}_{\mathrm{r}} \exp \left(\mathrm{ik}_{\mathrm{z}} \mathrm{z}\right) \mathrm{J}_{1}\left(\mathrm{k}_{\mathrm{r}} \mathrm{r}\right) \\
& \mathrm{E}_{\phi}=\mathrm{k}_{\mathrm{r}}^{2} \mathrm{~A}_{\phi} \exp \left(\mathrm{ik}_{\mathrm{z}} \mathrm{z}\right) \mathrm{J}_{1}\left(\mathrm{k}_{\mathrm{r}} \mathrm{r}\right) \\
& \mathrm{E}_{\mathrm{z}}=\mathrm{ik}_{\mathrm{z}} \mathrm{k}_{\mathrm{r}} \mathrm{A}_{\mathrm{z}} \exp \left(\mathrm{ik}_{\mathrm{z}} \mathrm{z}\right) \mathrm{J}_{0}\left(\mathrm{k}_{\mathrm{r}} \mathrm{r}\right)
\end{aligned}
$$

and

$$
\begin{gathered}
E_{r}=\omega \mu k_{z} A_{\phi} \exp \left(i k_{z} z\right) J_{1}\left(k_{r} r\right) \\
E_{\phi}=i \omega \mu k_{z} A_{r} \exp \left(i k_{z} z\right) J_{1}\left(k_{r} r\right) \\
E_{z}=i \omega \mu k_{r} A_{\phi} \exp \left(i k_{z} z\right) J_{0}\left(k_{r} r\right)
\end{gathered}
$$

They represent Bessel light beams with polarizations different from those of Table 1.

Let us now come back to the conventional Bessel beams made of nondiffractive waves defined in terms of the Bessel function $\mathrm{J}_{0}$. Concentrate around the propagation axis, their transverse shape is patterned by $\mathbf{J}_{0}$. Thus, these beams can be described as a bullseye surrounded by an infinite (finite for a quasi Bessel beam) number of concentrating rings.

So, when people, working with lasers, generate a beam, nondiffractive on some propagation distance, with an annular ring structure [7], their claim to have produced a Bessel beam could be an abuse of language since it is not proved that their annular rings have the representation $\mathrm{J}_{0}$.

\section{REFERENCES}

[1] Durnin J. (1987) Exact solutions for nondiffracting beams, J.Opt.Soc.Am.A 7, pp. 651-654.

[2] Durnin J. Miceli J.J, Eberly J.H., Diffraction free beams. (1987) Phys.Rev.Lett.52 pp.1499-1501.

[3] Sheppard C.(2001) Bessel pulse beams and focus wave modes, J.Opt.Soc.Am. A 18 pp.2594-2600.

[4] Hall D. Greene P.L. (1996) Diffraction characteristics of the azimuthal Bessel-Gauss beams.J.Opt.Soc.Am.A 13, pp.962-964.

[5] Hernandez-Figueroa H.E , Zamboni-Rached M, Recami E, (2008) Localized Waves, Wiley :Hoboken.

[6] Hernandez-Figueroa H.E., Zamboni-Rached M., Recami E. (2013) Non diffraacting waves.Wiley ,Hoboken

[7] Dudley A. Lavery M. Padgett M. Forbes A.(2013) Unraveiling Bessel beams, Optics and photonics 6 pp. 22 - 29.

[8] Bouchal Z, Olivik M. (1995) Nondiffractive vector Bessel beams, J.Mod.Opt.42 , pp. $1555=1566.1566$.

[9] Girgel S.S, Kurilkina S.N, (2001) Vector properties of Bessel light beams, Proc SPIE 358 Optics and Crystals :258.

[10] Yu Y.Z. .Dou W.B, (2008) Vector analysis of nondiffracting beams, PIER Letter .57 pp.71.

[11] Morse P.M., Feshbach H.,(1953) Methods of Theoretical Physics . Mc Graw Hill, New York.

[12] Volke-Sepulveda K., Garcès-Chavez V., Charvez-Cerda S. Arlt J. .Dholakia K.. (2002) Orbital angular momentumof high order Bessel light beams .J.Opt.Soc.Am B .4, pp.582-589 
International Journal of Recent advances in Physics (IJRAP) Vol.3, No.2, May 2014

[13] Jackson J.D.(1975 ) Classical Electromagnetism. Wiley : Hoboken..

[14] Stratton A. (1941) Electromagnetic Theory . Mc Graw Hill : New York ..

[15] Bouchal Z., Clechovsky R. Swartzland G.A. Spatially localized vortex structure.Chapter 13 in [4].

[16] Jones D.S, (1986) Acoustic aned Electromagnetic Waves. Clarendon Press : Oxford.

[17] Ren Z. Wu Q. Mao H. Shi Y. Fan C. (2013) Propagation characteristics of Airy Bessel wave packets in free space. Optics Express 21 pp. 481493.

[18] Hillion P. Electromagnetic beams and Hertz vectors. unpublished. 